\title{
Tecnologia digital versus convencional para confecção de próteses totais: uma
}

\section{revisão sistemática}

\author{
Digital versus conventional technology for making Complete Denture: a systematic review
}

Tecnología digital versus tecnología convencional para hacer dentaduras postizas totales: una revisión sistemática

Recebido: 11/01/2022 | Revisado: 16/01/2022 | Aceito: 22/01/2022 | Publicado: 24/01/2022

\author{
Larissa Costa Freitas \\ ORCID: https://orcid.org/0000-0002-6412-7073 \\ Universidade Federal de Juiz de Fora, Brasil \\ E-mail: larissacostafreitas@gmail.com \\ Flávia Braga de Oliveira \\ ORCID: https://orcid.org/0000-0003-0834-5743 \\ Universidade Federal de Juiz de Fora, Brasil \\ E-mail:flaviabraga.dentista@gmail.com \\ Camila Moreira Lima \\ ORCID: https://orcid.org/0000-0003-3233-5684 \\ Universidade Federal de Juiz de Fora, Brasil \\ E-mail:camila_moreira21@hotmail.com \\ Jean Soares Miranda \\ ORCID: https://orcid.org/0000-0001-5379-0155 \\ Universidade Federal de Juiz de Fora, Brasil \\ E-mail:jean.miranda@ufjf.br \\ Fabíola Pessôa Pereira Leite \\ ORCID: https://orcid.org/0000-0001-6316-5679 \\ Universidade Federal de Juiz de Fora, Brasil \\ E-mail:fabiola-leite1@ hotmail.com
}

\begin{abstract}
Resumo
Objetivo: Avaliar se a tecnologia CAD/CAM é superior a técnica convencional para confecção de próteses totais mucosuportadas. Metodologia: Esta revisão seguiu as recomendações do PRISMA. Foi realizada, por dois revisores calibrados, uma revisão sistemática nas bases de dados Medline-PubMed, Scopus, Scielo, Lilacs, Web of Science e Cochrane Library, usando os descritores: "Complete denture", "Complete dentures", "Total denture", "Total dentures", "Complete prosthesis", "Complete prostheses", "Total prosthesis", "Total prostheses", "Computer Aided Design", "Computer Aided Manufacturing", "CAD/CAM", "Benefits" e "Advantage". Foram incluídos apenas estudos in vitro que comparavam a tecnologia CAD/CAM (impressão ou fresagem) com a confecção convenvional e excluídos trabalhos sem grupo controle e de próteses sobre implantes. Resultados: A estratégia utilizada resultou em 410 artigos. Destes, 11 se encaixavam nos critérios de inclusão. Após leitura na íntegra, apenas 6 foram incluídos na revisão sistemática. Apesar de exigir um alto investimento e capacitação profissional, os estudos selecionados demonstraram as seguintes vantagens do sistema CAD/CAM sob o convencional: menor liberação de monômero e melhor adaptação, das próteses Conclusão: O processo de fabricação de prótese total por meio do sistema CAD/CAM apresentou-se vantajoso, comparado ao método convencional, sendo a principal delas a menor liberação de monômero, acompanhado de melhores qualidades biomecânicas e adaptação marginal, apesar do seu alto custo.
\end{abstract}

Palavras-chave: CAD-CAM; Prótese total; Vantagem.

\begin{abstract}
Objective: To assess whether the CAD/CAM technology is superior to the conventional technique for making Tissue Born total dentures. Methodology: This review followed the PRISMA recommendations. A systematic review was carried out by two calibrated reviewers in the Medline-PubMed, Scopus, Scielo, Lilacs, Web of Science and Cochrane Library databases, using the descriptors: "Complete denture", "Complete dentures", "Total denture", "Total dentures", "Complete prosthesis", "Complete prosthesis", "Total prosthesis", "Total prosthesis", "Computer Aided Design", "Computer Aided Manufacturing", "CAD/CAM", "Benefits" and "Advantage". Only in vitro studies comparing $\mathrm{CAD} / \mathrm{CAM}$ technology (printing or milling) with conventional manufacturing were included, and studies without a control group and prostheses on implants were excluded. Results: The strategy used resulted in 410 articles. Of these, 11 met the inclusion criteria. After reading in full, only 6 were included in the systematic review. Despite requiring a high investment and professional training, the selected studies demonstrated the following advantages of the CAD/CAM
\end{abstract}


system over the conventional one: less monomer release and better adaptation of the dentures Conclusion: The manufacturing process of total dentures through the system CAD/CAM proved to be advantageous compared to the conventional method, the main one being the lower release of monomer, accompanied by better biomechanical qualities and marginal adaptation, despite its high cost.

Keywords: CAD-CAM; Complete denture; Advantage.

\section{Resumen}

Objetivo: Evaluar si la tecnología CAD / CAM es superior a la técnica convencional para hacer dentaduras postizas totales con soporte de moco. Metodología: Esta revisión siguió las recomendaciones de PRISMA. Dos revisores calibrados realizaron una revisión sistemática en las bases de datos Medline-PubMed, Scopus, Scielo, Lilacs, Web of Science y Cochrane Library, utilizando los descriptores: "Dentadura completa", "Dentadura completa", "Dentadura total", "Prótesis total", "Prótesis completa", "Prótesis completa", "Prótesis total", "Prótesis total", "Diseño asistido por computadora", "Fabricación asistida por computadora", "CAD / CAM", "Beneficios" y "Ventaja". Solo se incluyeron los estudios in vitro que comparaban la tecnología CAD / CAM (impresión o fresado) con la fabricación convencional, y se excluyeron los estudios sin grupo control y prótesis sobre implantes. Resultados: La estrategia utilizada resultó en 410 artículos. De estos, 11 cumplieron los criterios de inclusión. Después de la lectura completa, solo se incluyeron 6 en la revisión sistemática. A pesar de requerir una alta inversión y formación profesional, los estudios seleccionados demostraron las siguientes ventajas del sistema CAD / CAM sobre el convencional: menor liberación de monómero y mejor adaptación de las prótesis Conclusión: El proceso de fabricación de prótesis totales mediante el sistema CAD / La CAM demostró ser ventajosa en comparación con el método convencional, siendo la principal la menor liberación de monómero, acompañada de mejores cualidades biomecánicas y adaptación marginal, a pesar de su elevado coste.

Palabras clave: CAD-CAM; Dentadura total; Ventaja.

\section{Introdução}

O uso da tecnologia CAD/CAM (Computer-aided design / Computer-aided manufacturing) difundil-se amplamente na Odontologia nas últimas décadas, permitindo ao Cirurgião-Dentista confeccionar próteses fíxas em consulta única, através da mescla de recursos como a velocidade e facilidade de manuseio, diminuindo a interferência do operador com um processo clínico e laboratorial simples e possibilitando uma longevidade reabilitadora (Sotto Maior et al., 2018; Joda e Brägger, 2016; Ludovichetti et al., 2018; Hwang et al., 2019). Esse sistema também permite a confecção de próteses removíveis baseado em um sistema tridimensional que possui o objetivo de automatizar, agilizar e controlar os processos de fabricação, minimizando a possibilidade de erros (Goodacre et al., 2016), pois permite a confecção de próteses removíveis com menor rugosidade, porosidade, o que contribui para menor liberação de monômeros e possibilita uma melhor higienização, além de propriciar um menor volume de resina, o que aumentam a sensação de conforto do paciente (Goodacre et al., 2016; Srinivasan et al., 2018). Por fim, uma grande vantagem é a obtenção de um arquivo digital que possibilita, caso necessário, a reimpressão rápida do modelo e da base da prótese (Goodacre et al., 2016; Srinivasan et al., 2018; Tavares et al., 2019).

Para obtenção de sucesso em uma reabilitação com próteses totais diversos fatores devem estar presentes, tais como, reprodução dos detalhes do rebordo com precisão e fidelidade dos modelos de trabalho (Atieh et al., 2017). O processo de confecção de próteses totais por meio do sistema CAD/CAM apresenta melhor acurácia (Melo et al., 2020), quando comparado ao método convencional, que exige uma ampla sequência de procedimentos clínicos e laboratoriais humano dependentes como moldagens, confecção de moldeira individual, montagem dos dentes, dentre outros (Paixão et al., 2007), isso porque as possíveis inconsistências das moldagens convencionais, que podem estar relacionadas com uma má preparação do material de moldagem e do gesso, levam muitas das vezes à necessidade de repetições, resultados clínicos e estéticos inferiores, além de aumentar a necessidade da realização de ajustes em boca (Yuzbasioglu et al., 2014; Koulivand et al. 2019).

Visto isso, a principal proposta desse estudo foi realizar uma revisão sistemática avaliando as vantagens, como a menor liberação de monômero, da utilização da tecnologia CAD/CAM sobre a metodologia convencional para confecção de próteses totais removíveis mucosuportadas (PTs) para pacientes edêntulos. A hipótese nula do presente estudo foi que a tecnologia CAD/CAM não contribui para uma melhor confecção de próteses totais, independentemente de ser através da impressão ou fresagem quando comparada a técnica convencional. 


\section{Metodologia}

Essa revisão foi redigida seguindo as recomendações do critério PRISMA (Preferred Reporting Items for Systematic Reviews and Meta-analyzes) (Moher et al., 2015), a pesquisa dos artigos foi realizada em maio de 2021 e inclui publicações dentre os anos 2010 e 2021.

\section{Critérios de elegibilidade}

Os pesquisadores adotaram suas análises com base no índice PICO, portanto: a população foi formada de pacientes edêntulos totais; a intervenção era a confecção, instalação e utilização de próteses totais removíveis mucosuportadas; a comparação foi realizada com as PTs confeccionadas através da metodologia convencional; e o desfecho foi a observação das vantagens daquela sobre essa última.

\section{Critério de inclusão de estudos}

Foram incluídos estudos in vitro que atenderam aos seguintes critérios: avaliação das vantagens de próteses totais confeccionadas por CAD/CAM; estudos que obtinham grupo(s) experimentais (fluxo CAD/CAM) e controle (fluxo convencional); estudos que utilizaram a tecnologia de impressão e/ou fresagem para avaliar próteses totais CAD/CAM. Foram excluídos estudos que não atenderam esses critérios, além dos que envolveram a confecções das próteses removíveis parciais, fixas e/ou sobre implantes.

\section{Estratégias de busca}

A estratégia de busca eletrônica foram realizadas por duas pesquisadoras (LCF e FBO) de forma independente entre janeiro e fevereiro de 2021, utilizando as bases de dados Pubmed, Medline, Web of Science, Scopus, LILACS e Scielo e os seguintes descritores: "Complete denture", "Complete dentures", "Total denture", "Total dentures", "Complete prosthesis", "Complete prostheses", "Total prosthesis", "Total prostheses", "Computer Aided Design", "Computer Aided Manufacturing", "CAD/CAM", "Benefits" e "Advantage". De acordo com esses descritores, as estratégias foram adequadas para cada tipo de base de dados (Tabela1). 
Tabela 1. Estratégias de buscas eletrônicas.

\begin{tabular}{ll}
\hline \multicolumn{1}{c}{ Base de dados } & \multicolumn{1}{c}{ Estratégias de buscas } \\
\hline PUBMED & $((((((($ Complete denture) OR (Complete dentures)) OR (Total denture)) OR (Total dentures)) \\
& OR (Complete prosthesis)) OR (Complete prostheses)) OR (Total prosthesis)) OR (Total \\
prostheses) $)$ AND ((Computer Aided Design) OR (Computer Aided Manufacturing) $)$ OR \\
(CAD/CAM)) AND ((Benefits) OR (Advantage)) \\
ALL("Complete denture" OR "Complete dentures" OR "Totaldenture" OR "Total dentures" OR \\
"Complete prosthesis" OR "Complete prostheses" OR "Total prosthesis" OR "Total prostheses") \\
AND ALL("Computer Aided Design" OR "Computer Aided Manufacturing" OR "CAD/CAM") \\
AND ALL("Benefits OR "Advantage") \\
TS=(Complete denture OR Complete dentures OR Total denture OR Total dentures OR \\
Complete prosthesis OR Complete prostheses OR Total prosthesis OR Total prostheses) AND \\
TS=(Computer Aided Design OR Computer Aided Manufacturing OR CAD/CAM) AND \\
TS=(Benefits OR Advantage) \\
(Complete denture OR Complete dentures OR Total denture OR Total dentures OR Complete \\
prosthesis OR Complete prostheses OR Total prosthesis OR Total prostheses) AND (Computer \\
Aided Design OR Computer Aided Manufacturing OR CAD/CAM) AND (Benefits OR \\
Advantage) \\
(Complete denture OR Complete dentures OR Total denture OR Total dentures OR Complete \\
prosthesis OR Complete prostheses OR Total prosthesis OR Total prostheses) AND (Computer \\
Aided Design OR Computer Aided Manufacturing OR CAD/CAM) AND (Benefits OR \\
Advantage) \\
(Complete denture OR Complete dentures OR Total denture ORTotal dentures OR Complete \\
prosthesis OR Complete prostheses OR Total prosthesis OR Total prostheses) AND \\
(Computer Aided Design OR Computer Aided Manufacturing OR CAD/CAM) AND(Benefits \\
OR Advantage) \\
\end{tabular}

Fonte: Autores (2021).

\section{Seleção de Estudos e Coleta de Dados}

Seguidamente à busca nas bases de dados, os títulos e resumos obtidos foram organizados de forma padronizada em uma planilha do Word (Office 2016, Microsoft, Redmond, Washington, EUA) manualmente. Foram removidos os artigos duplicados e em seguida as duas pesquisadoras, de forma independente, realizaram a análise de títulos/resumo para selecionar os estudos com potencial de serem lidos na íntegra. Em um próximo momento, realizou-se a leitura completa de todos os artigos na área, selecionando os estudos de acordo com os critérios de elegibilidade propostos.

Ao fim da leitura dos artigos na íntegra, alguns foram incluídos ou excluídos na revisão realizado pelas duas mestrandas (LCF e FBO). Reuniões foram realizadas para consenso da determinação da seleção dos artigos. Quando havia alguma discordância na busca entre as revisoras, essas eram assessoradas por uma terceira revisora doutoranda (CML).

\section{Processo de coleta de dados}

Dados qualitativos dos estudos incluídos na revisão foram extraídos a fim de caracterizar a análise descritiva (autor e ano do estudo, referências no decorrer do artigo, localização que o estudo foi realizado, tipo de estudo, grupos de estudo, amostragem, os parâmetros de avaliação das vantagens de próteses totais CAD/CAM sobre as convencionais e resultados relevantes) (Quadro 1). 
Quadro 1. Características e resumo dos resultados dos estudos incluídos na revisãosistemática

\begin{tabular}{|c|c|c|c|c|c|c|}
\hline Estudo & Local & Grupos & $\begin{array}{c}\text { Total de } \\
\text { amostras } \\
\text { (amostras por } \\
\text { grupos) } \\
\end{array}$ & $\begin{array}{l}\text { Parâmetros de } \\
\text { avaliação }\end{array}$ & Testes realizados & Resultados \\
\hline $\begin{array}{c}\text { Masri et al, } \\
2020\end{array}$ & Líbano & $\begin{array}{l}\text { PTs Convencionais; } \\
\text { fresadas e } \\
\text { impressas. }\end{array}$ & $60(20)$ & Adaptação da base & $\begin{array}{c}\text { Técnica de subtração } \\
\text { digital em } 5 \text { áreas } \\
\text { funcionais (selagem } \\
\text { palatina posterior, selagem } \\
\text { da borda anterior, crista da } \\
\text { crista, tuberosidades } \\
\text { maxilares) }\end{array}$ & $\begin{array}{c}\text { A técnica de fresagem } \\
\text { proporcionou uma melhor } \\
\text { adaptação da base ao rebordo } \\
\text { em todas as regiões } \\
\text { analisadas. }\end{array}$ \\
\hline $\begin{array}{l}\text { Steinmassl } \\
\text { et al. } 2016\end{array}$ & Áustria & $\begin{array}{l}\text { 4 sistemas } \\
\text { CAD/CAM: Baltic } \\
\text { Denture System, } \\
\text { Whole You Nexteeth, } \\
\text { Whole You Inc., } \\
\text { Wieland Digital } \\
\text { Dentures, Vita } \\
\text { VIONIC; e 1 grupo de } \\
\text { PTs convencionais }\end{array}$ & $50(10)$ & $\begin{array}{l}\text { Peso, volume, } \\
\text { densidade, calculo da } \\
\text { área de superfície, } \\
\text { liberação de } \\
\text { monômero resuduais }\end{array}$ & $\begin{array}{l}\text { Peso e volume da prótese } \\
\text { foram medidos. A } \\
\text { densidade foi calculada e a } \\
\text { área de superfície foi } \\
\text { avaliada digitalmente. } \\
\text { Cromatografia líquida de } \\
\text { alta eficiência. } \\
\text { Cromatografia gasosa }\end{array}$ & $\begin{array}{l}\text { Whole You Nexteeth e } \\
\text { Wieland Digital Dentures } \\
\text { apresentaram volume e peso } \\
\text { menores que a prótese } \\
\text { convencional.Baltic Denture } \\
\text { System apresentava menor } \\
\text { área de superfície que a } \\
\text { convencional; As PTs } \\
\text { CAD/CAM liberaram menos } \\
\text { monômero do que o grupo de } \\
\text { controle. }\end{array}$ \\
\hline $\begin{array}{l}\text { Einarsdottir } \\
\text { et al., } 2020\end{array}$ & EUA & $\begin{array}{l}3 \text { técnicas } \\
\text { diferentes: } \\
\text { convencional, } \\
\text { impressão e } \\
\text { fresagem } \\
\text { CAD/CAM } \\
\end{array}$ & $45(15)$ & $\begin{array}{l}\text { Estabilidade } \\
\text { dimensional }\end{array}$ & $\begin{array}{l}\text { Método de escaneamento e } \\
\text { sobreposição digital com o } \\
\text { modelo mestre em titânio } \\
\text { em } 22 \text { locais. }\end{array}$ & $\begin{array}{l}\text { As bases fresadas em } \\
\text { CAD/CAM têm menores } \\
\text { alterações dimensionais do } \\
\text { que a as demais avaliadas. }\end{array}$ \\
\hline $\begin{array}{l}\text { Ayman } \\
2017\end{array}$ & $\begin{array}{l}\text { Arábia } \\
\text { Saudita }\end{array}$ & $\begin{array}{c}\text { Dois grupos: PTs } \\
\text { convencionais e } \\
\text { CAD/CAM fresadas }\end{array}$ & $70(35)$ & $\begin{array}{l}\text { Resistência à flexão, a } \\
\text { dureza superficial e o } \\
\text { teor de monômero } \\
\text { residual na base da } \\
\text { prótese }\end{array}$ & $\begin{array}{l}\text { Teste Instron, teste de } \\
\text { dureza com aparelho } \\
\text { digital Micromet } \\
\text { Cromatografia gasosa }\end{array}$ & $\begin{array}{c}\text { As PTs CAD/CAM } \\
\text { apresentaram maior } \\
\text { resistência à flexão e baixo } \\
\text { valor do módulo de flexão. A } \\
\text { liberação do teor de } \\
\text { monômero foi maior no } \\
\text { grupo convencional. Mas a } \\
\text { dureza superficial do grupo } \\
\text { convencional foi } \\
\text { significativamente maior. }\end{array}$ \\
\hline $\begin{array}{l}\text { McLaughli } \\
\text { n } 2019\end{array}$ & EUA & $\begin{array}{c}3 \text { grupos: } \\
\text { CAD/CAM, } \\
\text { método por } \\
\text { compressão einjeção }\end{array}$ & $243(81)$ & $\begin{array}{l}\text { Moldagem da base de } \\
\text { cada PT em silicone e } \\
\text { verificação de } \\
\text { assentamento em um } \\
\text { modelo mestre de } \\
\text { titânio. } \\
\end{array}$ & $\begin{array}{c}\text { Avaliação assentamento da } \\
\text { prótese. }\end{array}$ & $\begin{array}{c}\text { CAD/CAM e } \\
\text { injeção apresentaram melhor } \\
\text { adaptação, prinpalmente para } \\
\text { palatos rasos. }\end{array}$ \\
\hline $\begin{array}{l}\text { Wemken et } \\
\text { al., } 2020\end{array}$ & $\begin{array}{l}\text { Alemanh } \\
\text { a }\end{array}$ & $\begin{array}{l}3 \text { grupos: PTs } \\
\text { impressas por } \\
\text { injeção (IM), } \\
\text { fresadas (MIL) e } \\
\text { estereolitografia } \\
\text { (SLA) }\end{array}$ & 48(16) & $\begin{array}{c}\text { Avaliação } \\
\text { assentamento da base } \\
\text { da PT, e das a } \\
\text { veracidade da } \\
\text { fabricação e precisão } \\
\text { IM, MIL e SLA; } \\
\text { impacto do } \\
\text { envelhecimento após o } \\
\text { envelhecimento } \\
\text { hidrotérmico e do } \\
\text { procedimento de } \\
\text { desinfecção }\end{array}$ & $\begin{array}{c}\text { Aposição, ciclagem } \\
\text { hidrotérmica e esterilização } \\
\text { por microondas. }\end{array}$ & $\begin{array}{l}\text { A fresagem possibilitou } \\
\text { melhor assentamento, } \\
\text { seguida por IM e SLA. O } \\
\text { envelhecimento hidrotérmico } \\
\text { apenas não afetou MIL. E } \\
\text { após a desinfecção em micro- } \\
\text { ondas, apenas SLA } \\
\text { permaneceram estáveis em } \\
\text { sua precisão e adaptação. }\end{array}$ \\
\hline
\end{tabular}

Fonte: Autores (2021).

\section{Avaliação da qualidade dos estudos e risco de viés}

O nível de evidência dos artigos selecionados foi classificado de acordo com o Oxford Center for Evidence-Based Medicine (Howick et al., 2011). Os estudos foram categorizados nos seguintes níveis: 1A, 1B, 1C, 2A, 2B, 2C, 3A, 3B, 4 e 5. Já a avaliação em relação ao desenho da metodologia e risco de viés foi feito por meio da Avaliação de Classificação de Recomendações, Sistema de Desenvolvimento e Avaliação (GRADE) (Howick et al., 2011) (Tabela 2). 
Tabela 2. Classificação do nível de evidência por tipo de estudo.

\begin{tabular}{lccc}
\hline Estudo & Ano doestudo & $\begin{array}{c}\text { Grau de } \\
\text { recomendação }\end{array}$ & Nível de evidência \\
\hline Masri et al & 2020 & $\mathrm{~B}$ & $2 \mathrm{~B}$ \\
\hline Steinmassl et al & 2016 & $\mathrm{~B}$ & $2 \mathrm{~B}$ \\
\hline Einarsdottir et al & 2020 & $\mathrm{D}$ & $2 \mathrm{C}$ \\
\hline Ayman & 2017 & $\mathrm{D}$ & $2 \mathrm{C}$ \\
\hline McLaughlin et al & 2019 & & $2 \mathrm{C}$ \\
\hline Wemken et al & 2020 & $\mathrm{~B}$ & $2 \mathrm{~B}$ \\
\hline
\end{tabular}

Fonte: Autores (2021).

\section{Resultados}

A lógica de busca eletrônica utilizada resultou em 410 artigos. Após a aplicação dos filtros estabelecidos, apenas 11 artigos foram selecionados e lidos na íntegra. Desses, apenas 7 atendiam aos critérios de elegibilidade e compuseram essa revisão sistemática. Nesses estudos eleitos foram avaliadas 516 bases de próteses, variando seus modos de confecção e avaliação de acordo com o artigo selecionado (Figura 1).

Figura 1. Diagrama de seleção de artigos para a revisão sistemática.

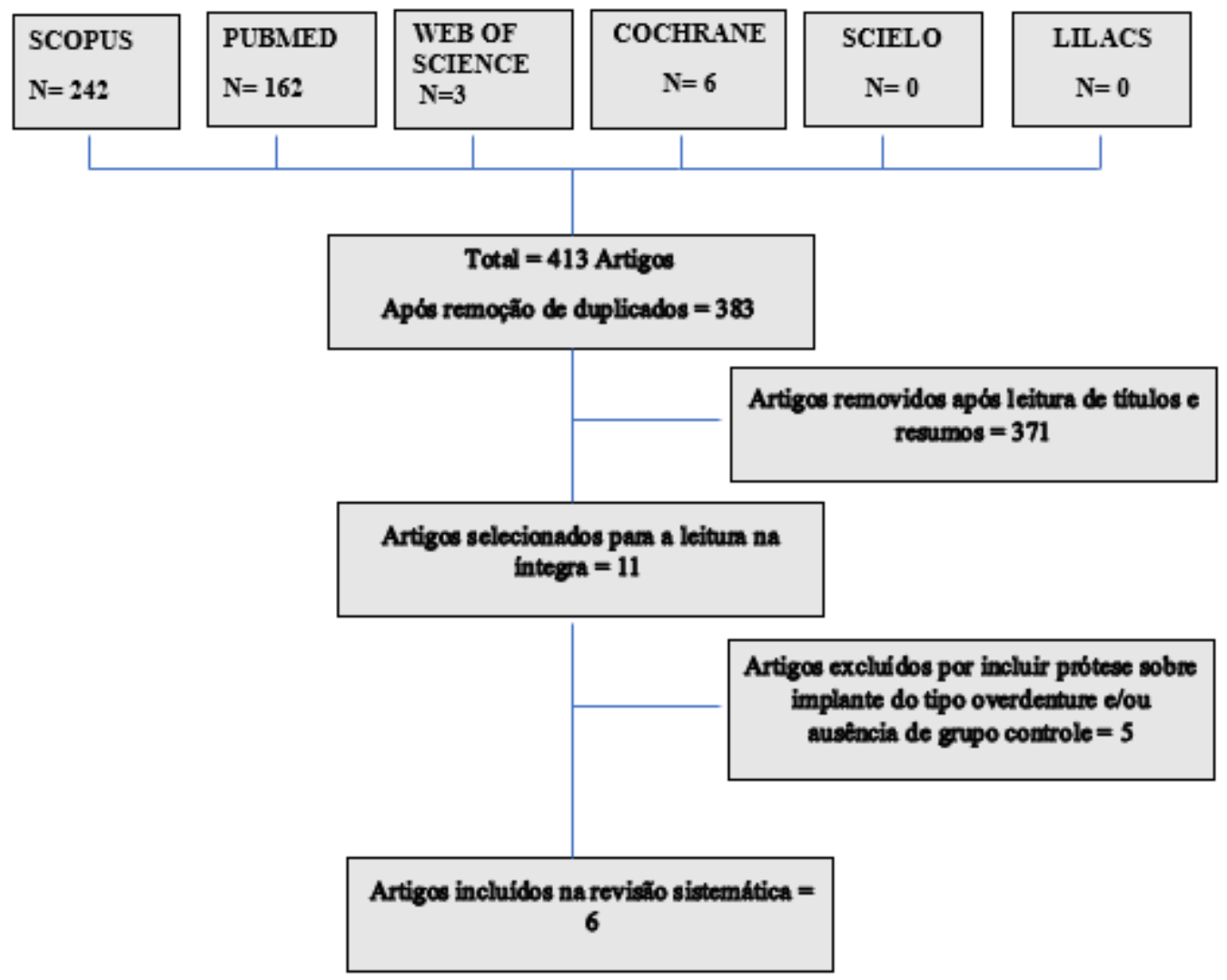

Fonte: Autores (2021). 
Pode-se observar que $85,7 \%$ obtiveram resultados satisfatórios quanto a liberação de monômero em relação às próteses confeccionadas pelo sistema CAD/CAM, incluindo as tecnologias de fresagem e impressão 3D,comparado com as confecções convencionais (Tabela 2).

\section{Discussão}

Todos os estudos que criteriosamente compuseram essa revisão sistemática expõem que, apesar de seu custo mais elevado, as próteses totais CAD/CAM apresentam alguma vantagem, em maior ou menor grau, independente do seu modo de obtenção (fresagem ou impressão), sobre as obtidas pelo método de sacralização convencional, o que leva a negação da hipótese nula desse estudo. Dentre essas vantagens destacam-se o menor volume de resina e menor peso, que aumentam o conforto do paciente (Steinmassl et al., 2016), além de maior dureza superficial (Ayman, 2017). A própria obtenção do modelo diminui as chances de ocorrer alterações dimensionais, visto que a moldagem convencional pode causar compressão contra o tecido pelo material de moldagem (Einarsdottir et al.,2020). A liberação de monômero residual também é menor para próteses CAD/CAM do que para as convencionais (Ayman et al., 2017), mesmo que nem sempre apresentem um valor significativamente menor (Steinmassl et al., 2016).

McLaughlin et al. (2019) investigaram a adaptação de bases confeccionadas pela técnica CAD/CAM fresadas e pela convencional ao palato. Eles observaram que a qualidade dessa adaptação foi diferente para cada material em função da anatomia dessa estrutura. Enquanto a técnica convencional apresentou bons resultados em palatos ovoides, a tecnologia CAD/CAM mostrou-se mais adaptativa em palatos rasos (McLaughlin et al., 2019). Um um estudo realizado por Steinmassl et al. (2018) o PMMA CAD/CAM demonstrou possuir uma superfície mais hidrofílica do que o convencional, o que contribui para a retenção da prótese (Steinmassl et al., 2018). Além disso, PMMA CAD/CAM tem melhor resistência à flexão e maior resistência à tração em comparação com PMMA convencionais (Ayman, A.D,2017).

Wemken et al. (2020) concluíram que, dentre as técnicas CAD/CAM, a fresagem das bases resulta em maior precisão do que impressão, técnica essa que é superior estereolitografia. Em seu estudo, observaram que a ciclagem hidrotérmica não foi prejudicial para as próteses fresadas, no entanto quando esterilizadas por micro-ondas, apenas as bases de próteses impressas com estereolitografia permaneceram dimensionalmente estáveis. Outros estudos (Masri et al., 2020; McLaughlin et al., 2019) também afirmam que a técnica de fresagem resulta em melhor adaptação e selamento em relação ao método impresso. Tal fato, pode ser explicado pela estabilidade do material industrialmente fabricado para ser fresado, que já se encontra polimerizado e não irá sofrer nenhuma deformação ou reestruturação de suas partículas (Masri et al., 2020, McLaughlin et al., 2019).

Apesar das várias vantagens da tecnologia CAD/CAM, deve-se destacar que as próteses totais obtidas pela técnica convencional apresentam significativa maior resistência à flexão e baixo valor do módulo de flexão em comparação com o material base de prótese de resina acrílica CAD/CAM (Ayman, 2017). Além disso, o fluxo digital exige um investimento alto, para o cirurgião-dentista e para o paciente (Srinivasan et al.,2020), requer uma curva de aprendizado quanto: aos softwares utilizados (Tavares et al., 2019), identificação da correta angulação da impressão para obtenção de um melhor assentamento (Vasques e Laganá, 2018), registro da dimensão vertical de oclusão e transferência da relação intermaxilar (Bilgin et al., 2016; Tavares et al., 2019). Alguns sistemas não possibilitam a correta definição do plano oclusal (Bilgin et al., 2016; Tavares et al., 2019), e também dificultam a realização de uma avaliação estética e fonética. Por fim, os materiais para impressão ou fresagem dificultam ajustes, como reembasamentos, já que a resina acrilíca convencional normalmente utilizada nesses reparos não é compatível com eles (Goodacre et al., 2016).

Além disso, a tecnologia CAD-CAM independente do seu modo de obtenção, seja por impressão ou fresagem, ainda não permite uma padronização absoluta pois a contribuição humana está envolvida no processo de fabricação (Steinmassl et al., 
2016), visto que os escaneamentos precisam ser digitalizados e convertidos em modelos virtuais edêntulos com um protocolo de digitalização de impressão de laboratório durante a fabricação da prótese (Peng et al., 2018).

É válido justificar que nessa revisão foram incluídos apenas estudos laboratoriais in vitro devido a ausência de estudos do tipo ensaio clínico randomizado que realizaram essa comparação, o que infelizmente proporciona um nível menor de confiabilidade. Os estudos selecionados foram avaliados e classificados de acordo com seu nível de evidência cientifica seguindo o Oxford Center for Evidence-Based Medicine para garantir a validade, similaridade e reprodutibilidade dos estudos (Howick et al., 2011). Outra limitação dessa revisão sistemática é não incluir uma metanálise, no entanto, apesar de alguns estudos apresentarem metodologias similares, no geral houve uma ausência de homogeneidade metodológica nos parâmetros de avaliação da adaptaçãodas próteses. Nesse sentido, uma investigação mais aprofundada das propriedades mecânicas dos materiais CAD/CAM é necessária para avaliar outras propriedades ainda não relatados nesse artigo, além da nexessidade de realização de ensaios clínicos com esses materiais.

\section{Considerações Finais}

Foi possível concluir a partir dos estudos que a confecção de próteses totais removíveis mucosuportadas obtidas através da tecnologia CAD/CAM apresentam vantagens sobre a técnica convencional, destacando-se por: menor liberação de monômero, menor peso, densidade, maior dureza e melhor adaptação aos tecidos de suporte. No entanto, novos matérias para confecção dessas vem surgindo constantemente e, portanto, novos estudos laboratoriais e clínicos devem ser constantemente realizados para conhecer suas propriedades.

\section{Referências}

Atieh M.A, Ritter A.V., Ko C.C.\& Duqum I. (2017). Accuracy evaluation of intraoral optical impressions: A clinical study using a reference appliance. J Prosthet Dent. 118(3):400-5.

Ayman A.D. (2017). The residual monomer content and mechanical properties of CAD \CAM resins used in the fabrication of complete dentures as compared to heat cured resins. Electron Physician. 25;9(7):4766-72.

Baba, N. Z., Goodacre, C. J., \& Kattadiyil, M. T. (2015). CAD/CAM removable prosthodontics. Masri R, Driscoll CF. Clinical applications of digital dental technology. Hoboken, 107-10.

Bilgin, M. S., Baytaroğlu, E. N., Erdem, A., \& Dilber, E. (2016). A review of computer-aided design/computer-aided manufacture techniques for removable denture fabrication. European journal of dentistry, 10(2), 286.

Goodacre, B. J., Goodacre, C. J., Baba, N. Z. \& Kattadiyil, M. T. (2016). Comparison of denture base adaptation between CAD-CAM and conventional fabrication techniques. J Prosthet Dent, 116(2), 249-256.

Howick, J., Chalmers, I., Glasziou, P., Greenhalgh, T., Heneghan, C., Liberati, A., et al. (2011). The 2011 Oxford CEBM Evidence levels of evidence (introductory document). Oxford Center for Evidence-Based Medicine, 1-3.

Hwang, H. J., Lee, S. J., Park, E. J. \& Yoon, H. I. (2019). Assessment of the trueness and tissue surface adaptation of CAD-CAM maxillary denture bases manufactured using digital light processing. J Prosthet Dent, 121(1), 110-117.

Joda, T. \& Brägger, U. (2016). Patient-centered outcomes comparing digital and conventional implant impression procedures: a randomized crossover trial. Clinical Oral Implants Research, 27(12), 185-89.

Koulivand S.; Ghodsi S.; Siadat H. \& Alikhasi M. (2019). A clinical comparison of digital and conventional impression techniques regarding finish line locations and impression time. Journal of esthetic and restorative dentistry. Disponível em: https://onlinelibrary.wiley.com/doi/10.1111/jerd.12527.

Ludovichetti F.S., Trindade F.Z., Werner A., Kleverlaan C.J.\& Fonseca R.G. (2018). Wear resistance and abrasiveness of CAD-CAM monolithic materials. $J$ Prosthet Dent. Aug;120(2):318.e1-318.e8.

Masri, G., Mortada, R., Ounsi, H., Alharbi, N., Boulos, P. \& Salameh, Z. (2020). Adaptation of Complete Denture Base Fabricated by Conventional, Milling, and 3-D PrintingTechniques: An In Vitro Study. J Contemp Dent Pract, 21(4), 367-371.

McLaughlin, J. B., Ramos Jr., V. \& Dickinson, D. P. (2019). Comparison of Fit of Dentures Fabricated by Traditional Techniques Versus CAD/CAM Technology. J Prosthodont, 28(4), 428-435.

Melo, L.A.; Lima C.M; Ferrarez L.L.; Silva D.N. Leite F.P.P. (2020). Precisão da adaptação de próteses totais feitas com a tecnologia CAD / CAM: uma revisão sistemática. Pesquisa, Sociedade e Desenvolvimento, 9(10), n. 10, e6839109154. 
Research, Society and Development, v. 11, n. 2, e24911225625, 2022

(CC BY 4.0) | ISSN 2525-3409 | DOI: http://dx.doi.org/10.33448/rsd-v11i2.25625

Moher D., Shamseer L., Clarke M., Ghersi D., Liberati A., Petticrew M., Shekelle P.\& Stewart L.A. Declaração de 2015 dos itens de relatório preferidos para protocolos de revisão sistemática e meta-análise (PRISMA-P). Syst Rev. 2015; 4 (1): 1. 10.1186 / 2046-4053-4-1

Paixão, F., Ramos, G. D. G., \& Cruz, M. V. D. J. (2007). Evaluation of the reproducibility of two techniques used to determine and record centric relation in angle's class I patients. Journal of Applied Oral Science, 15(4), 275-279.

Peng L., Chen L., Harris B.T., Bhandari B., Morton D.\& Lin W.S. (2018). Accuracy and reproducibility of virtual edentulous casts created by laboratory impression scan protocols. J Prosthet Dent. Sep. 120(3):389-395.

Sotto Maior B.S, Filgueiras A., Gonçalves Pinto D., Ferrarez L. L., de Oliveira M. F.\& Freitas T.A. de C. (2018). Aplicabilidade clínica dos avanços da tecnologia CAD-CAM em Odontologia. hu rev;44(1):29-34.

Steinmassl, O., Dumfahrt, H., Grunert, I., \& Steinmassl, P. A. (2018). Influence of CAD/CAM fabrication on denture surface properties. Journal of oral rehabilitation, 45(5), 406-413.

Steinmassl P.A., Wiedemair V., Huck C., Klaunzer F., Steinmassl O., Grunert I. \& Dumfahrt H. (2017). Do CAD/CAM dentures really release less monomer than conventional dentures? Clin Oral Investig. Jun. 21(5):1697-705.

Srinivasan, M., Kalberer, N., Naharro, M., Marchand, L., Lee, H., \& Müller, F. (2020). CAD-CAM milled dentures: The Geneva protocols for digital dentures. The Journal of prosthetic dentistry, 123(1), 27-37.

Srinivasan, M., Gjengedal, H., Cattani-Lorente, M., Moussa, M., Durual, S., Schimmel, M. \& Müller, F. (2018). CAD/CAM milled complete removable dental prostheses: An in vitro evaluation of biocompatibility, mechanical properties, and surface roughness. Dent Mater J, 37(4), 526-533.

Tavares, C. C., Freire, J. C. P., Freire, S. C. P., Dias-Ribeiro, E., \& Batista, A. U. D. (2019). Aplicabilidade dos sistemas CAD/CAM em Prótese Total: revisão de literatura. Arch Health Invest, 7(11).

Vasques, M. T., \& Laganá, D. C. (2018). Accuracy and Internal Fit of 3D printed Occlusal Splint, according to the printing position: A technique report. Clinical and Laboratorial Research in Dentistry.

Wemken G., Spies B.C., Pieralli S., Adali U., Beuer F. \& Wesemann C. (2020). Do hydrothermal aging and microwave sterilization affect the trueness of milled, additive manufactured and injection molded denture bases? J Mech Behav Biomed Mater. 111:103975.

Yuzbasioglu E., Kurt H., Turunc R. \& Bilir H. (2014). Comparison of digital and conventional impression techniques: evaluation of patients' perception, treatment comfort, effectiveness and clinical outcomes. BMC Oral Health30; 14:10. 